\title{
Moons, bridges, birds ... and nonexpansive mappings in Hilbert space
}

\section{Kasimierz Goebel and Rainald Schöneberg}

\begin{abstract}
In recent years some fixed point theorems have been proved for nonexpansive mappings in Hilbert space, which have non-convex domains. The purpose of this paper is to present a simple but very useful new result of that kind and to indicate some of its consequences.
\end{abstract}

\section{Introduction}

Throughout our discussion $H$ will denote a Hilbert space, $X$ will denote a nonempty bounded subset of $H$, and for a subset $Y$ of $H$ we use $\overline{c o n v}(Y)$ to denote the convex closure of $Y$. A mapping $f: X \rightarrow H$ such that $|f(x)-f(y)| \leq|x-y|$ for all $x, y \in X$, is called nonexpansive. $X$ is said to have the fixed point property for nonexpansive mappings, if every nonexpansive self-map of $X$ has a fixed point.

The precise generality of the class of sets having the fixed point property for nonexpansive mappings is not known, but it does include all closed bounded and convex sets ([2], [3], [5]), all contractible finite unions of closed bounded and convex sets ([6]), and all closed bounded and starshaped sets ([4]).

In this short note we prove - among others - that a non-empty bounded subset of $H$ has the fixed point property for nonexpansive mappings, if it is Chebyshev with respect to its convex closure. Thus we establish the fixed point property for nonexpansive mappings for several sets which are not necessarily weakly closed or starshaped, and get a useful tool for

Received 8 August 1977. 
constructing a lot of irregular sets having the fixed point property for nonexpansive mappings .

\section{Main result}

THEOREM. Let $f: X \rightarrow X$ be nonexpansive. Then the following properties of $f$ are equivalent:

(1) $f$ has a fixed point in $X$;

(2) there is $x_{0} \in X$ such that for any

$$
y \in \overline{\operatorname{conv}}\left\{\left\{f^{n}\left(x_{0}\right): n \in z^{+}\right\}\right\}
$$

there is a unique $x<X$ such that $|y-x|=\operatorname{dist}(y, X)$.

Proof. (1) $\Rightarrow(2)$ : obvious. (2) $\Rightarrow(1)$ : In view of Kirszbraun's Theorem ([7]) there is a nonexpansive mapping $g: H \rightarrow \overline{\operatorname{conv}}(X)$ such that $\left.g\right|_{X}=f$. Then the sequence $\left(\frac{1}{n+1} \sum_{k=0}^{n} g^{k}\left(x_{0}\right)\right)$ converges we'zkly to a fixed point $y$ of $g([1])$. Since $g^{k}\left(x_{0}\right)=f^{k}\left(x_{0}\right)$ for $k \in z^{+}$, we have $y \in \overline{\operatorname{conv}}\left\{\left\{f^{n}\left(x_{0}\right): n \in z^{+}\right\}\right\}$, and hence there is a unique $x \in X$ such that $|y-x|=\operatorname{dist}(y, x)$. Then $\operatorname{dist}(y, x) \leq|y-f(x)|=|g(y)-g(x)| \leq|y-x|=\operatorname{dist}(y, x) ;$

that is, $|y-f(x)|=\operatorname{dist}(y, X)$, and therefore $x=f(x)$.

\section{Some consequences}

1. Let $S=\{x \in H:|x|=1\}$ and $f: S \rightarrow S$ be nonexpansive. Then $f$ has a fixed point in $S$ iff there is $x_{0} \in S$ such that

$0 \notin \overline{\operatorname{conv}}\left\{\left\{f^{n}\left(x_{0}\right): n \in z^{+}\right\}\right\}$.

2. Let $X$ be Chebyshev with respect to its convex closure (that is, for any $y \in \overline{\operatorname{conv}}(X)$ there is a unique $x \in X$ such that $|y-x|=\operatorname{dist}(y, x))$. Then $X$ has the fixed point property for nonexpansive mappings.

3. Let $x^{*}$ be a linear functional of unit norm on $H$ and 
$0<c<1$. Then 2 implies that the set

$$
\left\{x \in H:|x|=1 \text { and } r e x^{*}(x) \geq c\right\}
$$

has the fixed point property for nonexpansive mappings. This has been established in [8], if $\sqrt{15} / 4<c<1$.

4. The following (two-dimensional) figures indicate further (infinite-dimensional) subsets of $H$, which have the fixed point property for nonexpansive mappings by 2 :
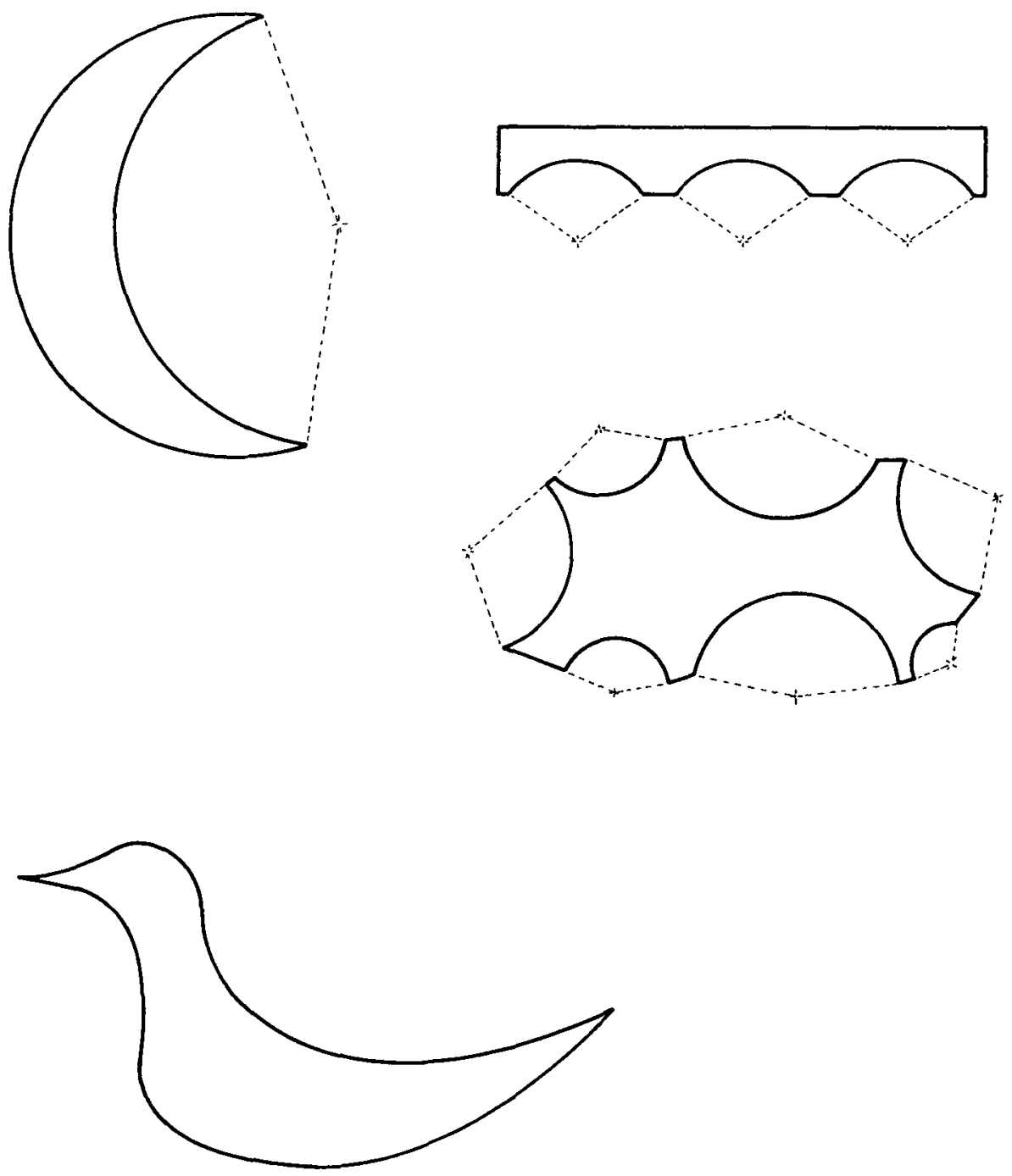


\section{Two problems}

PROBLEM 1. Does there exist a convex body $Y$ in $H$ such that the boundary of $Y$ is Chebyshev with respect to its convex closure?

PROBLEM 2. Does there exist a convex body $Y$ in $H$ such that the boundary of $Y$ has the fixed point property for nonexpansive mappings?

\section{References}

[1] Jean-Bernard Baillon, "Un théereme de type ergodique pour les contractions non linéaires dans un espace de Hilbert", C.R. Acad. Sci. Paris Ser. A 280 (1975), 1511-1514.

[2] Felix E. Browder, "Nonexpansive nonlinear operators in a Banach space", Proc. Nat. Acad. Sci. U.S.A. 54 (1965), 1041-1044.

[3] Dietrich Göhde, "Zum Prinzip der kontraktiven Abbildung", Math. Nachr. 30 (1965), 251-258.

[4] Dietrich Göhde, "Elementare Bemerkungen zu nichtexpansiven Selbstabbildungen nicht konvexer Mengen im Hilbertraum", Math. Nachr. $63(1974), 331-335$.

[5] W.A. Kirk, "A fixed point theorem for mappings which do not increase distances", Amer. Math. Monthly 72 (1965), 1004-1006.

[6] J. Reinermann and R. Schöneberg, "Some results in fixed point theory for nonexpansive and pseudocontractive mappings in Hilbertspace", Fixed point theory and its applications, 187-196 (Proc. Seminar Fixed Point Theory and its Applications, Dalhousie University, Halifax, 1975. Academic Press [Harcourt Brace Jovanovich], New York, San Francisco, London, 1976).

[7] 1.J. Schoenberg, "On a theorem of Kirzbraun and Valentine", Amer. Math. Monthly 60 (1953), 620-622.

[8] John Staples, "Fixed point theorems in uniformly rotund metric spaces", Bul2. Austral. Math. Soc. 14 (1976), 181-192.

Instytut Matematyki, Uniwersytet Mari i Curie - Sklodowskiej, Lubl in, Poland;
Lehrstuhl C für Mathematik, Rheinisch-Westfälische Technische Hochschule, Aachen, Germany. 